\title{
Characterization of homogeneous symmetric monotone bivariate means
}

\author{
Mustapha Raïssouli ${ }^{1,2^{*}}$ (D) and Anis Rezgui ${ }^{1,3}$
}

\section{${ }^{*}$ Correspondence:}

raissouli.mustapha@gmail.com

'Department of Mathematics,

Science Faculty, Taibah University,

P.O. Box 30097, Al Madinah

Al Munawwarah, 41477, Saudi

Arabia

${ }^{2}$ Department of Mathematics,

Science Faculty, Moulay Ismail

University, Meknes, Morocco

Full list of author information is

available at the end of the article

\begin{abstract}
In this paper, we introduce a class of bivariate means generated by an integral of a continuous increasing function on $(0,+\infty)$. This class of means widens the spectrum of possible means and leads to many easy and interesting mean-inequalities. We show that this class of means characterizes the large class of homogeneous symmetric monotone means.
\end{abstract}

MSC: $26 \mathrm{E} 60$

Keywords: bivariate mean; differential equation; intrinsic function

\section{Introduction}

In recent few years, many authors have introduced plenty of classes of bivariate means in terms of functions or integrals of functions; see, for instance, [1-5]. Most of these classes of means require very specific assumptions and/or conditions on the function, which in fact restricts the range of the underlined class of means. In this paper, we also introduce a class of bivariate means defined as an integral of functions, where the underlying function is just continuous and increasing on $(0, \infty)$, which is not restrictive and so gives much more possibilities to define new means and new mean-inequalities.

The main result of the paper is a characterization result; in fact, we show that our class of means characterizes a large class of means, the class of homogeneous symmetric monotone means.

The paper is organized as follows: Section 2 is devoted to the introduction of the new class of means with some examples. In Section 3, we focus ourselves on comparison results, that is, on how we could obtain mean-inequalities of interest using this new formulation. In the last section, we prove a characterization result; in fact, we show that every homogeneous symmetric strict monotone mean can be seen as an element of our class of means.

Before starting the next section, let us recall some basic definitions and examples about bivariate means. By a (bivariate) mean we understand a function $m$ defined on $(0, \infty) \times$ $(0, \infty)$ that satisfies the following double inequality:

$$
\forall a, b>0, \quad \min (a, b) \leq m(a, b) \leq \max (a, b)
$$

A mean is said to be strict if the inequalities in (1.1) are both strict for all $a \neq b$. A continuous (resp. symmetric/homogeneous) mean is defined as usual; see, for instance, [6].

(c) 2016 Raïssouli and Rezgui. This article is distributed under the terms of the Creative Commons Attribution 4.0 International License (http://creativecommons.org/licenses/by/4.0/), which permits unrestricted use, distribution, and reproduction in any medium, provided you give appropriate credit to the original author(s) and the source, provide a link to the Creative Commons license, and indicate if changes were made. 
Standard examples of such means are the following:

$$
\begin{aligned}
& A:=A(a, b)=\frac{a+b}{2} ; \quad G:=G(a, b)=\sqrt{a b} ; \quad H:=H(a, b)=\frac{2 a b}{a+b} ; \\
& S:=S(a, b)=\left(\frac{\sqrt{a}+\sqrt{b}}{2}\right)^{2} ; \quad L:=L(a, b)=\frac{b-a}{\ln b-\ln a}, \quad L(a, a)=a ; \\
& Q:=Q(a, b)=\sqrt{\frac{a^{2}+b^{2}}{2}} ; \quad C:=C(a, b)=\frac{a^{2}+b^{2}}{a+b},
\end{aligned}
$$

which are known in the literature as the arithmetic mean, geometric mean, harmonic mean, square-root mean, logarithmic mean, quadratic (or root-square) mean, and contraharmonic mean, respectively. For more examples and details about bivariate means and their applications, we refer the reader to [7] and the references therein.

An interesting example of nonsymmetric homogeneous mean is the so-called SchwabBorchardt mean, denoted by $S B$, and defined as $[8,9]$

$$
S B:=S B(a, b)= \begin{cases}\frac{\sqrt{b^{2}-a^{2}}}{\cos ^{-1}(a / b)} & \text { if } 0<a<b, \\ \frac{\sqrt{a^{2}-b^{2}}}{\cosh ^{-1}(a / b)} & \text { if } a>b,\end{cases}
$$

with $S B(a, a)=a$. This nonsymmetric mean stems its importance in the fact that it includes a lot of symmetric means in the sense that

$$
L=S B(A, G), \quad P=S B(G, A), \quad T=S B(A, Q), \quad M=S B(Q, A),
$$

where

$$
\begin{aligned}
& P:=P(a, b)=\frac{a-b}{4 \tan ^{-1}(\sqrt{a / b})-\pi}=\frac{a-b}{2 \sin ^{-1}\left(\frac{a-b}{a+b}\right)}=\frac{a-b}{4 \tan ^{-1}\left(\frac{\sqrt{a}-\sqrt{b}}{\sqrt{a}+\sqrt{b}}\right)}, \quad P(a, a)=a ; \\
& T:=T(a, b)=\frac{a-b}{2 \tan ^{-1}\left(\frac{a-b}{a+b}\right)}=\frac{a-b}{2 \tan ^{-1}(a / b)-\pi / 2}=\frac{a-b}{\sin ^{-1}\left(\frac{a^{2}-b^{2}}{a^{2}+b^{2}}\right)}, \quad T(a, a)=a ; \\
& M:=M(a, b)=\frac{a-b}{2 \sinh ^{-1}\left(\frac{a-b}{a+b}\right)}, \quad M(a, a)=a ;
\end{aligned}
$$

they are, respectively, known as the first Seiffert mean [10], the second Seiffert mean [11], and the Neuman-Sándor mean [8]. For more details about recent developments for $S B$, see, for instance, $[8,9,12]$. The previous standard means satisfy the well-known chain of inequalities

$$
H<G<L<P<A<M<T<Q \text {, }
$$

where the notation $m_{1}<m_{2}$, between two means $m_{1}$ and $m_{2}$, means that $m_{1}(a, b)<m_{2}(a, b)$ for all $a, b>0$ with $a \neq b$.

For a homogeneous mean $m$, we define its associate function $\phi_{m}:(0, \infty) \longrightarrow(0, \infty)$ via the relationship $m(a, b)=b m(a / b, 1)=b \phi_{m}(a / b)$, that is, $\phi_{m}(x)=m(x, 1)$ for all $x>0$. In this case, (1.1) yields

$$
x \leq \phi_{m}(x) \leq 1 \quad \text { if } 0<x \leq 1, \quad 1 \leq \phi_{m}(x) \leq x \quad \text { if } x \geq 1 .
$$


A homogeneous mean $m$ is called monotone if $\phi_{m}$ is increasing on $(0, \infty)$. In what follows, if there is no confusion, then we write $\phi$ instead of $\phi_{m}$. The means $A, G, H, S, Q$, and $L$ are monotone, whereas $C$ is not; see [6] for more details.

\section{A new class of bivariate means}

As mentioned before, in the sequel, we introduce and investigate a new class of means generated by an integral of a function. Let $f$ be a continuous strictly monotonic function on the open interval $(0, \infty)$. For every $a, b>0$, we define

$$
m_{f}(a, b)=\frac{b}{f(a / b)-f(1)} \int_{f(1)}^{f(a / b)} f^{-1}(u) d u \quad \text { with } m_{f}(a, a)=a .
$$

Proposition 2.1 For any continuous and strictly monotonic function $f$ on $(0,+\infty)$, the binary map $m_{f}$ defined by (2.1) is a homogeneous bivariate mean.

Proof It is straightforward and therefore omitted here.

Remark 2.1 The mean $m_{f}$ is not always symmetric; see Example 2.3 and Example 2.2. Otherwise, it is not hard to check that $m_{f+c}=m_{f}$ for each constant $c$ and that $m_{\alpha, f}=$ $m_{f}$ for every $\alpha \neq 0$. In particular, $m_{-f}=m_{f}$. Due to this, without loss of generality, we only consider functions $f$ that are continuous strictly increasing on $(0, \infty)$ and satisfying $f(1)=0$.

In the sequel, we will use the following notations:

$$
C_{\uparrow}^{0}(0, \infty)=\{f:(0, \infty) \longrightarrow f(0, \infty): f \text { is continuous and strictly increasing }\}
$$

and

$$
C_{\uparrow}^{1}(0, \infty)=\left\{f \in C_{\uparrow}^{0}(0, \infty): f \text { is continuously differentiable }\right\} .
$$

The following result gives other equivalent forms of $m_{f}$.

Proposition 2.2 Let $f \in C_{\uparrow}^{0}(0, \infty)$ be such that $f(1)=0$. Then the following assertions hold:

(i) For all $a, b>0$, we have

$$
m_{f}(a, b)=b \int_{0}^{1} f^{-1}(t f(a / b)) d t
$$

(ii) If, moreover, $f$ is differentiable, then we have (for all $a, b>0, a \neq b$ )

$$
\begin{aligned}
& m_{f}(a, b)=\frac{b}{f(a / b)} \int_{1}^{a / b} u f^{\prime}(u) d u, \\
& m_{f}(a, b)=a-\frac{b}{f(a / b)} \int_{1}^{a / b} f(u) d u .
\end{aligned}
$$


Proof (i) If in (2.1) we make the change of variables $u=t f(a / b)$ with $0 \leq t \leq 1$, then we obtain (2.2) by simple topics of integration.

(ii) Setting $u=f(s)$ in (2.1), we obtain (2.3) after an elementary manipulation. By integration by parts, (2.4) follows from (2.3).

The previous forms of $m_{f}$ lead to the following regularity result.

Corollary 2.3 Let $f \in C_{\uparrow}^{0}(0, \infty)$ be such that $f(1)=0$. Then the mean $m_{f}$ is continuous strict monotone.

Proof Since $f$ is continuous and $m_{f}$ is a mean, the continuity of $m_{f}$ follows from (2.1) with standard topics of real analysis. The fact that $m_{f}$ is strict monotone follows from (2.2). The details are simple and therefore omitted here.

We now present some examples that illustrate the previous mean $m_{f}$. The first example shows that the mean $m_{f}$ includes the standard means $A, H, G$, and $L$.

\section{Example 2.1}

(i) With $f(x)=x-1$, we easily verify that $m_{f}=A$. If $f(x)=\ln x$, then $m_{f}=L$. Letting $f(x)=1-1 / \sqrt{x}$, simple computation leads to $m_{f}=G$, and if $f(x)=1-1 / x^{2}$, then $m_{f}=H$.

(ii) Let $f(x)=-1 / x+1$. By (2.4) simple computation leads to (for $a, b>0, a \neq b$ )

$$
m_{f}(a, b)=\frac{a b}{b-a}(\ln b-\ln a)=\frac{a b}{L(a, b)}:=L^{*}(a, b),
$$

which is the dual logarithmic mean, that is, $L^{*}(a, b)=\left(L\left(a^{-1}, b^{-1}\right)\right)^{-1}$.

We now state the following example, which, in its turn, includes a lot of the most interesting standard means.

Example 2.2 Let $f$ be defined by $f(x)=x^{p}-x^{q}$, where $p \geq 0, q \leq 0$, and $(p, q) \neq(0,0)$. It is easy to see that $f \in C_{\uparrow}^{1}(0, \infty)$ with $f(1)=0$. By (2.3), elementary computation leads to (after all manipulations and reductions)

$$
m_{f}(a, b)=\frac{p(q+1) b^{q}\left(a^{p+1}-b^{p+1}\right)+q(p+1) b^{p}\left(a^{q+1}-b^{q+1}\right)}{(p+1)(q+1)\left(a^{p} b^{q}-a^{q} b^{p}\right)}:=W_{p, q}(a, b)
$$

for all $a, b>0, a \neq b$, with $q \neq-1$. For $q=-1$, we have

$$
W_{p,-1}(a, b)=\lim _{q \rightarrow-1} W_{p, q}(a, b)=a\left(\frac{p}{p+1}+\frac{b^{p+1}(\ln a-\ln b)}{a^{p+1}-b^{p+1}}\right)
$$

and, in particular,

$$
W_{1,-1}(a, b)=\frac{1}{2}\left(a+\frac{b G^{2}}{A L}\right)
$$

which shows that $m_{f}$ is in general not symmetric. Following Corollary 2.3, $W_{p, q}(a, b)$ is continuous and strictly increasing in $a$ and $b$. The 2-power mean $W_{p, q}$ also includes 
another example of symmetric power mean in the sense that $W_{0, q}(a, b)=D_{q}(a, b)$ and $W_{p, 0}(a, b)=D_{p}(a, b)$, where $D_{r}$ refers to the so-called 1-power difference mean defined for all real number $r$ by

$$
\left\{\begin{array}{l}
D_{r}:=D_{r}(a, b)=\frac{r}{r+1} \frac{a^{r+1}-b^{r+1}}{a^{r}-b^{r}}, \quad D_{r}(a, a)=a, \\
D_{-2}=H, \quad D_{-1}=L^{*}, \quad D_{-1 / 2}=G, \quad D_{0}=L, \quad D_{1}=A .
\end{array}\right.
$$

Remark 2.2 Formula (2.3) remains true if $f$ is only differentiable on $(0,1) \cup(1, \infty)$, provided that the integral

$$
\int_{1}^{a / b} u f^{\prime}(u) d u
$$

is well-defined (i.e., convergent as an improper integral).

As already pointed in the Introduction, the Schwab-Borchardt mean $S B$ is of great interest since it includes a lot of symmetric means. Our approach includes, in turn, the mean $S B$. The following example explains this latter situation and that of the previous remark. It also shows that $m_{f}$ is not always symmetric.

Example 2.3 Let $f$ be defined by

$$
f(x)=-\cos ^{-1} x \quad \text { if } 0<x \leq 1, \quad f(x)=\cosh ^{-1} x \quad \text { if } x \geq 1 .
$$

Clearly, $f$ is continuous and strictly increasing on $(0, \infty)$ and differentiable on $(0,1) \cup$ $(1, \infty)$. By (2.3) and Remark 2.2 we have (for all $a, b>0$ with $a \neq b$ )

$$
m_{f}(a, b)= \begin{cases}\frac{b}{-\cos ^{-1}(a / b)} \int_{1}^{a / b} \frac{u}{\sqrt{1-u^{2}}} d u=\frac{\sqrt{b^{2}-a^{2}}}{\cos ^{-1}(a / b)} & \text { if } 0<a<b \\ \frac{b}{\cosh ^{-1}(a / b)} \int_{1}^{a / b} \frac{u}{\sqrt{u^{2}-1}} d u=\frac{\sqrt{a^{2}-b^{2}}}{\cosh ^{-1}(a / b)} & \text { if } a>b\end{cases}
$$

that is, $m_{f}=S B$. It is well-known that $S B(a, b)$ is continuous and strictly monotonic increasing in its variables $a$ and $b$. This is again immediately confirmed by the previous Corollary 2.3.

After discussing some examples, we are now in a position to state the following result, which shows that the mean map $f \longmapsto m_{f}$ is one-to-one modulus multiplication by positive real numbers.

Theorem 2.4 Let $f, g \in C_{\uparrow}^{1}(0, \infty)$ be such that $f(1)=g(1)=0$ and $g^{\prime}(1) \neq 0$. Then, $m_{f}=m_{g}$ if and only if $f=\alpha \cdot g$ for some real number $\alpha>0$.

Proof Assume that $m_{f}=m_{g}$, that is, $m_{f}(a, b)=m_{g}(a, b)$ for all $a, b>0$. By (2.4) we have (after simple manipulation)

$$
g(a / b) \int_{1}^{a / b} f(u) d u=f(a / b) \int_{1}^{a / b} g(u) d u
$$


for all $a, b>0$. Setting $x=a / b$, we then have

$$
g(x) \int_{1}^{x} f(u) d u=f(x) \int_{1}^{x} g(u) d u
$$

or, equivalently,

$$
\int_{1}^{x} f(u) d u=\frac{f(x)}{g(x)} \int_{1}^{x} g(u) d u
$$

for all $x \in(0,1) \cup(1, \infty)$. Differentiating (with respect to $x$ ) both sides of the last equality we obtain

$$
f(x)=\frac{f^{\prime}(x) g(x)-f(x) g^{\prime}(x)}{(g(x))^{2}} \int_{1}^{x} g(u) d u+\frac{f(x)}{g(x)} g(x)
$$

and then

$$
\left(f^{\prime}(x) g(x)-f(x) g^{\prime}(x)\right) \int_{1}^{x} g(u) d u=0
$$

for all $x \in(0,1) \cup(1, \infty)$. Since $g$ is strictly increasing on $(0, \infty)$, it is not hard to check that

$$
\forall x \in(0,1) \cup(1, \infty), \quad \int_{1}^{x} g(u) d u>0 .
$$

It follows that $f^{\prime}(x) g(x)-f(x) g^{\prime}(x)=0$ or, equivalently,

$$
\frac{f^{\prime}(x)}{f(x)}=\frac{g^{\prime}(x)}{g(x)}
$$

for all $x \in(0,1) \cup(1, \infty)$. By integrating both sides of this last equality, there exist two constants $\lambda_{1}$ and $\lambda_{2}$ such that

$$
\ln |f(x)|= \begin{cases}\ln |g(x)|+\lambda_{1} & \text { if } x \in(0,1) \\ \ln |g(x)|+\lambda_{2} & \text { if } x \in(1, \infty)\end{cases}
$$

We then deduce that

$$
f(x)=\left\{\begin{array}{ll}
c_{1} g(x) & \text { if } x \in(0,1), \\
c_{2} g(x) & \text { if } x \in(1, \infty),
\end{array} \quad \text { and also } \quad f^{\prime}(x)= \begin{cases}c_{1} g^{\prime}(x) & \text { if } x \in(0,1) \\
c_{2} g^{\prime}(x) & \text { if } x \in(1, \infty)\end{cases}\right.
$$

for some two constants $c_{1}$ and $c_{2}$. Since $f$ and $g$ are continuously differentiable on $(0, \infty)$, we must have

$$
f^{\prime}(1)=\lim _{x \rightarrow 1^{-}} f^{\prime}(x)=c_{1} g^{\prime}(1)=\lim _{x \rightarrow 1^{+}} f^{\prime}(x)=c_{2} g^{\prime}(1),
$$

which, with $g^{\prime}(1) \neq 0$, yields $c_{1}=c_{2}:=\alpha$. In summary, using the fact that $f(1)=g(1)=0$, we have showed that $f(x)=\alpha \cdot g(x)$ for all $x \in(0, \infty)$. Since $f$ and $g$ are both strictly increasing, we conclude that $f=\alpha \cdot g$ with $\alpha>0$. Conversely, if $f=\beta \cdot g$ for some $\beta>0$, then $m_{f}=$ $m_{\beta \cdot g}=m_{g}$. The proof is finished. 


\section{Mean-inequalities}

In the ongoing section we shall state some mean-inequalities that are either new or easy to obtain using the class of means introduced in the previous section. Despite its general interest in mathematical analysis, it remains true that the major interest of introducing new classes of means is the obtention of mean-inequalities.

Let us first recall some topics that will be needed later. The double inequality

$$
h\left(\frac{a+b}{2}\right) \leq \frac{1}{b-a} \int_{a}^{b} h(u) d u \leq \frac{h(a)+h(b)}{2}
$$

holds for every convex function $h:[a, b] \longrightarrow \mathbb{R}, a<b$. If $h$ is concave, then (3.1) are reversed. Such a double inequality is known in the literature as the Hermite-Hadamard inequality, (HHI) in short, and is important in mathematical analysis. For further details about (HHI) and some of its extensions, refinements, and applications, see, for instance, [13-16]. It is worth mentioning that if the function $h$ is strictly convex (resp. strictly concave), then (3.1) are strict (resp. strict and reversed).

Now, we may state the following propositions.

Proposition 3.1 Let $f \in C_{\uparrow}^{0}(0, \infty)$ be such that $f(1)=0$. Then the following assertions hold:

(i) Iff is convex, then for all $a, b>0$, we have

$$
\frac{a+b}{2} \leq m_{f}(a, b) \leq b f^{-1}\left(\frac{1}{2} f(a / b)\right)
$$

with reversed inequalities iff is concave.

(ii) Iff is convex and differentiable, then, for all $a, b>0, a \neq b$, we have

$$
\frac{a+b}{2} \leq m_{f}(a, b) \leq a-\frac{a-b}{f(a / b)} f\left(\frac{a+b}{2 b}\right)
$$

with reversed inequalities iff is concave.

Proof (i) Assume that $f$ is convex. Since $f$ is strictly increasing, then $f^{-1}$ is concave. By (3.1) with (2.1) we then have

$$
b \frac{f^{-1}(f(1))+f^{-1}(f(a / b))}{2} \leq m_{f}(a, b) \leq b f^{-1}\left(\frac{f(1)+f(a / b)}{2}\right)=b f^{-1}\left(\frac{1}{2} f(a / b)\right)
$$

from which the desired double inequality follows.

(ii) follows by combining (2.4) with (3.1).

Similarly, (2.3) with (3.1) immediately yields the following result.

Proposition 3.2 Let $f \in C_{\uparrow}^{0}(0, \infty)$ be differentiable such that $f(1)=0$. Assume further that the map $u \longmapsto u f^{\prime}(u)$ is convex on $(0, \infty)$. Then, for all $a, b>0$, we have:

$$
\frac{a^{2}-b^{2}}{2 b f(a / b)} f^{\prime}\left(\frac{a+b}{2 b}\right) \leq m_{f}(a, b) \leq \frac{a-b}{2 b f(a / b)}\left(a f^{\prime}(a / b)+b f^{\prime}(1)\right)
$$


Remark 3.1 If in the previous results the considered functions are strictly convex (resp. strictly concave), then the associated inequalities are strict (resp. reversed).

Remark 3.2 Inequalities (3.4) become equalities for $f(u)=u-1$ and $g(u)=\ln u$, which correspond to $m_{f}=A$ and $m_{f}(a, b)=L(a, b)$, respectively. This is because the real function $u \longmapsto u f^{\prime}(u)$ is linear affine if and only if $f(u)=c_{1} u+c_{2} \ln u+c_{3}$ for some constants $c_{1}, c_{2}$, and $c_{3}$ to be chosen to ensure $f \in C_{\uparrow}^{0}(0, \infty)$ with $f(1)=0$, that is, $c_{1}, c_{2} \geq 0, c_{1}+c_{2} \neq 0$, and $c_{1}+c_{3}=0$.

We now present some examples that illustrate the previous results.

Example 3.1 Let $f(x)=\ln x$, which is strictly increasing and strictly concave on $(0, \infty)$. We have seen that $m_{f}=L$. By Proposition 3.1 and Remark 3.1, (3.2) and (3.3) are here reversed. After simple computation, (3.2) gives the known double inequality $G<L<A$. See also [3] for a direct way.

Example 3.2 Let $f(x)=x^{p}-1$, for which $m_{f}=D_{p}$ (see Example 2.2). If $0<p<1$, then $f \in C_{\uparrow}^{1}(0, \infty)$ and $x \longmapsto x f^{\prime}(x)$ is strictly concave on $(0, \infty)$. Then (3.4), with Remark 3.1, yields

$$
J_{p}(a, b):=\frac{p}{2}(a-b) \frac{a^{p}+b^{p}}{a^{p}-b^{p}}<D_{p}(a, b)<\frac{p}{2^{p}}(a-b) \frac{(a+b)^{p}}{a^{p}-b^{p}}:=K_{p}(a, b)
$$

for all $a, b>0$ with $a \neq b$. It is not hard to see that the two binary maps $J_{p}(a, b)$ and $K_{p}(a, b)$ are both means. In particular, simple computations yield $J_{1 / 2}=S$ and $K_{1 / 2}=A^{1 / 2} S^{1 / 2}$.

It appears to be interesting to determine convenient conditions on $f$ for which the leftand right-hand sides of (3.4) are binary means.

The next result shows that direct comparison of two functions $f, g \in C_{\uparrow}^{0}(0, \infty)$ leads to inequalities involving $m_{f}$ and $m_{g}$.

Proposition 3.3 Let $f, g \in C_{\uparrow}^{0}(0, \infty)$ be such that $f(1)=g(1)=0$. Assume further that $f$ and $g$ are both differentiable. Then the following assertions hold:

(i) If $f(x)<g(x)$ for all $x>0, x \neq 1$, then, for all $a, b>0, a \neq b$, we have

$$
(f(a / b))^{2}\left(a-m_{f}(a, b)\right)<(g(a / b))^{2}\left(a-m_{g}(a, b)\right)
$$

(ii) If $f^{\prime}(x) \leq g^{\prime}(x)$ for all $x>0$, then, for all $a, b>0$, we have

$$
|f(a / b)| m_{f}(a, b) \leq|g(a / b)| m_{g}(a, b) .
$$

Proof (i) Assume that $f(x)<g(x)$ for all $x>0, x \neq 1$, and let $a, b>0$ with $a \neq b$. Since $f(a / b)$ and $(a / b-1)$ have the same sign, we have

$$
\frac{b}{f(a / b)} \int_{1}^{a / b} f(x) d x<\frac{b}{f(a / b)} \int_{1}^{a / b} g(x) d x
$$


Since by assumption $f(a / b)<g(a / b)$ and $f, g$ are both increasing and have the same sign, we have $1 / g(a / b) \leq 1 / f(a / b)$. This, together with (3.5) and $\int_{1}^{a / b} f(x) d x>0$, yields

$$
\frac{b}{g(a / b)} \int_{1}^{a / b} f(x) d x \leq \frac{b}{f(a / b)} \int_{1}^{a / b} g(x) d x
$$

We can then conclude by (2.4) with a simple manipulation.

(ii) Denote

$$
F^{*}(y)=\int_{0}^{y} f^{-1}(u) d u, \quad G^{*}(y)=\int_{0}^{y} g^{-1}(u) d u .
$$

Since $f^{\prime}(x) \leq g^{\prime}(x)$ for all $x>0$, we have

$$
\begin{aligned}
\left(F^{*} \circ f\right)^{\prime}(x) & =\left(\int_{0}^{f(x)} f^{-1}(u) d u\right)^{\prime}=x f^{\prime}(x) \\
& \leq x g^{\prime}(x)=\left(\int_{0}^{g(x)} g^{-1}(u) d u\right)^{\prime}=\left(G^{*} \circ g\right)^{\prime}(x) .
\end{aligned}
$$

This, with the help of the classical mean value theorem, implies

$$
\forall x>0, x \neq 1, \quad \frac{F^{*} \circ f(x)}{x-1} \leq \frac{G^{*} \circ g(x)}{x-1}
$$

since $F^{*} \circ f(1)=G^{*} \circ g(1)=0$. It follows that

$$
\forall x>0, x \neq 1, \quad \frac{F^{*} \circ f(x)}{f(x)} \frac{f(x)}{x-1} \leq \frac{G^{*} \circ g(x)}{g(x)} \frac{g(x)}{x-1}
$$

or, equivalently,

$$
\forall a, b>0, a \neq b, \quad m_{f}(a, b) \leq \frac{g(a / b)}{f(a / b)} m_{g}(a, b),
$$

from which the desired result follows after simple manipulation. The proof is completed.

The following example illustrates the previous proposition.

Example 3.3 Let $f:(0, \infty) \longrightarrow(-\pi / 4, \pi / 4)$ be defined by

$$
f(x)=\arctan x-\frac{\pi}{4}=\arctan \frac{x-1}{x+1} .
$$

By (2.3) a simple computation of integral leads to (for all $a, b>0, a \neq b$ )

$$
m_{f}(a, b)=\frac{b\left(\ln \left(a^{2}+b^{2}\right)-2 \ln b-\ln 2\right)}{2 \arctan (a / b)-\pi / 2}:=\tilde{T}(a, b) .
$$

Let $g(x)=(1 / 2) \ln x$ for which $m_{g}=L$. It is clear that

$$
f^{\prime}(x)=1 /\left(1+x^{2}\right) \leq 1 /(2 x)=g^{\prime}(x)
$$


for all $x>0$. By Proposition 3.3(ii) we have that, for all $a, b>0, a \neq b$,

$$
|\arctan (a / b)-\pi / 4| \tilde{T}(a, b) \leq \frac{1}{2}|\ln a-\ln b| L(a, b)=\frac{|a-b|}{2}
$$

or, equivalently, $\tilde{T}(a, b) \leq T(a, b)$, where $T$ is the second Seiffert mean defined in the Introduction.

Proposition 3.4 Let $f, g \in C_{\uparrow}^{0}(0, \infty)$ be such that $f(1)=g(1)=0$. Then the following assertions are satisfied:

(i) If the composed function $g \circ f^{-1}$ is convex (resp. concave), then $m_{f} \leq m_{g}$ (resp. $\left.m_{f} \geq m_{g}\right)$.

(ii) Iff is concave and $g$ is convex, then $m_{f} \leq A \leq m_{g}$.

Proof (i) Assume that $g \circ f^{-1}$ is convex. Then we have

$$
g \circ f^{-1}(t f(a / b))=g \circ f^{-1}((1-t) f(1)+t f(a / b)) \leq(1-t) g(1)+\operatorname{tg}(a / b)=\operatorname{tg}(a / b) .
$$

If we apply $g^{-1}$ (which is strictly increasing) to both sides, then we get

$$
f^{-1}(t f(a / b)) \leq g^{-1}(\operatorname{tg}(a / b))
$$

which, by integration over $t \in(0,1)$ and the help of $(2.2)$, yields the desired result.

(ii) Let id be the identity map of $(0, \infty)$. If $f$ is concave (resp. $g$ is convex), then id $\circ f^{-1}$ is convex (resp. $g \circ \mathrm{id}^{-1}$ is convex). To conclude, we apply (i) knowing that $m_{\mathrm{id}-1}=A$.

Remark 3.3 If, in the previous proposition, we replace the word 'convex' (resp. 'concave') by 'strictly convex' (resp. 'strictly concave'), then all the related inequalities are strict.

The following example illustrates the previous result.

Example 3.4 Let $f(x)=\ln x$ and $g(x)=x-1 / x$. We have seen that $m_{f}=L$ and $m_{g}=W_{1,-1}$; see Example 2.2. For all $x>0$, we have $g \circ f^{-1}(x)=g\left(e^{x}\right)=2 \sinh x$, which is strictly convex on $(0, \infty)$. By Proposition 3.4(i) with Remark 3.3 we have $L<W_{1,-1}$. On the other hand, it is easy to see that $g$ is strictly concave on $(0, \infty)$, and by Proposition 3.4(ii), with Remark 3.3 again, we infer that $W_{1,-1}<A$. In summary, we have proved that $L<W_{1,-1}<A$. This double inequality, when combined with (2.5), yields

$$
L<\frac{1}{2}\left(a+\frac{b G^{2}}{A L}\right)<A
$$

From the left-hand side of the last double inequality we can deduce an inequality involving the three means $A, G$, and $L$. We omit details about this situation and refer the reader to Example 3.5 for a similar way.

The next corollary can be considered as an example of the previous proposition. 
Corollary 3.5 Let $f \in C_{\uparrow}^{0}(0, \infty)$ be such that $f(1)=0$. Assume that $f$ is bounded below on $(0, \infty)$, that is, for all $x>0, f(x)>-\alpha$ for some $\alpha>0$. Then we have:

$$
m_{\ln ((1 / \alpha) f+1)}<m_{f}<m_{\alpha(\exp f-1)}
$$

Proof Since $m_{f}=m_{(1 / \alpha) f}$, we can then assume that $\alpha=1$. Now, remark that $\ln (f+1)$ and $\exp f-1$ are strictly increasing on $(0, \infty)$ with $(\ln (f+1))(1)=(\exp f-1)(1)=0$. On one hand, we have

$$
(\exp f-1) \circ f^{-1}(x)=\exp \left(f \circ f^{-1}(x)\right)-1=e^{x}-1
$$

which is strictly convex. On the other hand, it is easy to verify that

$$
(\ln (f+1))^{-1}(x)=f^{-1}\left(e^{x}-1\right) \text { and so } f \circ(\ln (f+1))^{-1}(x)=e^{x}-1
$$

The desired double inequality then follows from Proposition 3.4(i) when combined with Remark 3.3.

We now state the following example explaining how to use the previous corollary. Although it is a very simple example, we will use it as a good tool for obtaining a symmetric homogeneous bivariate mean that appears to us to be new.

Example 3.5 Let $A=m_{f}$ with $f(x)=x-1>-1$ for all $x>0$. Then it is easy to see that $m_{\ln (f+1)}=L$ and

$$
\forall a, b>0, a \neq b, \quad m_{\exp f-1}(a, b)=\frac{a-b}{1-\exp (1-a / b)} .
$$

Applying Corollary 3.5, (3.6) turns to be

$$
\forall a, b>0, a \neq b, \quad L(a, b)<A(a, b)<\frac{a-b}{1-\exp (1-a / b)} .
$$

Now, set

$$
\forall a, b>0, a \neq b, \quad \alpha:=\alpha(a, b)=\frac{a-b}{1-\exp (1-a / b)} .
$$

The right-hand side of (3.7) gives $A(a, b)<\alpha(a, b)$, and by the symmetry of $A$ we also have $A(a, b)<\alpha(b, a)$. Let $H$ be the harmonic mean, which is strictly monotone. We then have

$$
A=H(A, A)<H(\alpha(a, b), \alpha(b, a))<\max (\alpha(a, b), \alpha(b, a))<\max (a, b)
$$

since $\alpha$ is a mean. Using the standard explicit form of $H:=H(a, b)=2\left(a^{-1}+b^{-1}\right)^{-1}$ for all $a, b>0$, we obtain by a simple computation (for all $a, b>0, a \neq b$ )

$$
H(\alpha(a, b), \alpha(b, a))=\frac{2(a-b)}{\exp (1-b / a)-\exp (1-a / b)} .
$$

Summarizing the previous discussion, we have the following result. 
Proposition 3.6 The binary map

$$
\forall a, b>0, \quad Z(a, b):=\frac{2(a-b)}{\exp (1-b / a)-\exp (1-a / b)}, \quad Z(a, a)=a,
$$

is a symmetric homogeneous mean satisfying $A<Z$.

\section{Characterization of homogeneous symmetric monotone means}

We preserve the same notation. For $f \in C_{\uparrow}^{0}(0, \infty)$, we have seen that $m_{f}$ is a continuous and strict monotone mean. Inversely, given a mean $m$, under what condition there exists $f \in C_{\uparrow}^{0}(0, \infty)$ such that $m=m_{f}$ ? We should note here that the answer of the latter question has to be seen as a characterization of bivariate means that could be fitted in the class of means introduced in (2.1).

Before giving an answer to that question, we state the following technical lemmas.

Lemma 4.1 Let $x_{0} \in \mathbb{R}$, and let $p, q$ be two continuous functions on $V \backslash\left\{x_{0}\right\}$ for some neighborhood $V$ of $x_{0}$. Assume that $p(x) \simeq q(x)$ as $x \rightarrow x_{0}$, that is, $\lim _{x \rightarrow x_{0}} p(x) / q(x)=1$, and $\lim _{x \rightarrow x_{0}} p(x)=\lim _{x \rightarrow x_{0}} q(x)= \pm \infty$. Then we have:

$$
\int p(x) d x \simeq \int q(x) d x \quad \text { as } x \rightarrow x_{0}
$$

where the notation $\int g(x) d x$ refers to the antiderivative of the function $g$.

Proof It is not hard to verify that the assumption

$$
\lim _{x \rightarrow x_{0}} p(x)=\lim _{x \rightarrow x_{0}} q(x)=+\infty \quad(\text { resp. }-\infty)
$$

implies

$$
\lim _{x \rightarrow x_{0}} \int p(x) d x=\lim _{x \rightarrow x_{0}} \int q(x) d x=+\infty \quad(\text { resp. }-\infty) .
$$

Now, to obtain the desired result, we just apply the classical L'Hopital rule.

Lemma 4.2 Let $m$ be a symmetric homogeneous mean, and $\phi$ its associate function. Then the following assertions hold:

(i) If $\phi$ is differentiable, then $\phi^{\prime}(1)=1 / 2$.

(ii) If $\phi$ is continuously differentiable then

$$
\lim _{x \rightarrow 1} \frac{(x-1) \phi^{\prime}(x)}{x-\phi(x)}=1
$$

(iii) If (4.1) holds, then we have

$$
\lim _{x \rightarrow 1} \exp \left(\int \frac{\phi^{\prime}(x)}{x-\phi(x)} d x\right)=0
$$


Proof (i) First, (1.4) immediately gives $\phi(1)=1$. Now, writing $m(x, 1)=x m(1,1 / x)=$ $x m(1 / x, 1)$, we then have $\phi(x)=x \phi(1 / x)$ for all $x>0$. This relation gives, by differentiation,

$$
\forall x>0, \quad \phi^{\prime}(x)+\frac{1}{x} \phi^{\prime}\left(\frac{1}{x}\right)=\phi\left(\frac{1}{x}\right)
$$

which, with $x=1$, yields $\phi^{\prime}(1)=1 / 2$.

(ii) It is easy to see that

$$
\lim _{x \rightarrow 1} \frac{(x-1) \phi^{\prime}(x)}{x-\phi(x)}=\lim _{x \rightarrow 1} \frac{\phi^{\prime}(x)}{\frac{x-1-(\phi(x)-1)}{x-1}}=\lim _{x \rightarrow 1} \frac{\phi^{\prime}(x)}{1-\frac{\phi(x)-\phi(1)}{x-1}}=\frac{\phi^{\prime}(1)}{1-\phi^{\prime}(1)}=1
$$

since $\phi$ is continuously differentiable and $\phi^{\prime}(1)=1 / 2$. This proves the desired result. We can also prove it by using L'Hopital's rule.

(iii) Applying Lemma 4.1 to (4.1) written in the next form

$$
\frac{\phi^{\prime}(x)}{x-\phi(x)} \simeq \frac{1}{x-1} \quad \text { when } x \rightarrow 1
$$

we then deduce

$$
\int \frac{\phi^{\prime}(x)}{x-\phi(x)} d x \simeq \int \frac{1}{x-1} d x=\ln |x-1|+\text { constant } \quad \text { as } x \rightarrow 1
$$

This implies that

$$
\lim _{x \rightarrow 1} \int \frac{\phi^{\prime}(x)}{x-\phi(x)} d x=-\infty
$$

and the desired result follows.

Lemma 4.3 Let $m$ be a symmetric homogeneous strict monotone mean, and $\phi$ its associate function. Assume that $\phi$ is continuously differentiable. Then the ordinary differential equation $(O D E)$

$$
\left\{\begin{array}{l}
(\phi(x)-x) y^{\prime}+\phi^{\prime}(x) y=0, \quad x \in(0,1) \cup(1, \infty) \\
y(1)=0
\end{array}\right.
$$

admits at least one solution in $C_{\uparrow}^{1}(0, \infty)$.

Proof Since $m$ is strict, the equation $\phi(x)=x$ has the unique solution $x=1$. Then the ODE (4.3) has to be solved separately on both intervals $(0,1)$ and $(1, \infty)$, and then we should show that the two solutions could be joined at $x=1$. The general solution of the ODE (4.3) can be written as

$$
y(x)= \begin{cases}y_{1}(x):=c_{1} \exp \left\{\int \frac{\phi^{\prime}(x)}{x-\phi(x)} d x\right\}, & 0<x<1, \\ y_{2}(x):=c_{2} \exp \left\{\int \frac{\phi^{\prime}(x)}{x-\phi(x)} d x\right\}, & x>1,\end{cases}
$$


where $c_{1}$ and $c_{2}$ are two arbitrary constants, to be conveniently chosen later. It is clear that

$$
y_{i}^{\prime}(x)=\frac{\phi^{\prime}(x)}{x-\phi(x)} y_{i}(x), \quad i=1,2 .
$$

Since $m$ is strict monotone, then $\phi^{\prime}(x)>0$ for all $x>0$. This, with Lemma 4.2, allows us to choose $c_{1}>0$ and $c_{2}<0$ to ensure $y_{i}^{\prime}(x)>0$ for $i=1,2$. Consider the particular solutions of ODE (4.3) such that $c_{1}=1$ and $c_{2}=-1$. Now, we will show that we can join the two solutions $y_{i}, i=1,2$. This means that

$$
\lim _{x \rightarrow 1^{-}} y_{1}(x)=\lim _{x \rightarrow 1^{+}} y_{2}(x)=0 .
$$

This immediately follows from (4.2) of Lemma 4.2 applied to (4.4). Let us denote by $y$ the piecewise function defined on $(0,+\infty)$ as follows:

$$
y(x)= \begin{cases}y_{1}(x) & \text { if } x \in(0,1), \\ 0 & \text { if } x=1, \\ y_{2}(x) & \text { if } x \in(1, \infty) .\end{cases}
$$

To end the proof, we wish to establish that our chosen solution $y$ is continuously differentiable on $(0, \infty)$. Obviously, $y$ is continuously differentiable on $(0,1) \cup(1, \infty)$. Further, following our choice with Lemma 4.2, we have $y(x) \simeq x-1$ as $x \rightarrow 1$, and so $y$ is differentiable at $x=1$ with $y^{\prime}(1)=1$. On the other hand, since

$$
\forall x>0, x \neq 1, \quad y^{\prime}(x)=\frac{\phi^{\prime}(x)}{x-\phi(x)} y(x)
$$

and by Lemma 4.2 again

$$
\frac{\phi^{\prime}(x)}{x-\phi(x)} \simeq \frac{1}{x-1} \quad \text { as } x \rightarrow 1
$$

we then deduce

$$
y^{\prime}(x) \simeq \frac{y(x)}{x-1} \quad \text { as } x \rightarrow 1 .
$$

This implies that $\lim _{x \rightarrow 1} y^{\prime}(x)=1=y^{\prime}(1)$ since $y(x) \simeq x-1$ as $x \rightarrow 1$. The proof is finished.

Now, we are in a position to state our characterization theorem, which answers the question stated at the beginning of this section.

Theorem 4.4 Let $m$ be a symmetric homogeneous strict monotone mean, and $\phi$ its associate function. Assume that $\phi$ is continuously differentiable. Then there exists $f_{m}:=f \in$ $C_{\uparrow}^{1}(0, \infty)$ with $f(1)=0$ such that $m=m_{f}$. Further, such $f$, called here the intrinsic function of $m$, is the solution of the ODE (4.3) in Lemma 4.3.

Proof According to Lemma 4.3, the ODE (4.3) has at least a solution in $C_{\uparrow}^{1}(0, \infty)$, which we denote here by $f$. We then have (for all $x>0$ )

$$
(\phi(x)-x) f^{\prime}(x)+\phi^{\prime}(x) f(x)=0
$$


with $f(1)=0$. It follows that

$$
\phi(x) f^{\prime}(x)+\phi^{\prime}(x) f(x)=x f^{\prime}(x)
$$

or, equivalently,

$$
(\phi f)^{\prime}(x)=x f^{\prime}(x)
$$

This yields

$$
\phi(x) f(x)=\int_{1}^{x} u f^{\prime}(u) d u .
$$

Since $f(1)=0$ and $f$ is strictly increasing, we have $f(x) \neq 0$ for each $x \neq 1$. We can then write, for all $x \neq 1$,

$$
\phi(x)=\frac{1}{f(x)} \int_{1}^{x} u f^{\prime}(u) d u,
$$

which, with (2.3) and $\phi(1)=1=m_{f}(1,1)$, gives $\phi(x)=m_{f}(x, 1)$ for all $x>0$. The desired result follows since $m$ and $m_{f}$ are homogeneous.

Before giving some examples illustrating the previous theoretical study, we would like to interpret the previous theorem in another point of view. For this, let us denote by $\mathcal{M}_{\text {shsm }}$ the set of all symmetric homogeneous strict monotone means and by $\mathcal{F}$ the set of all $f \in$ $C_{\uparrow}^{1}(0, \infty)$ such that $f(1)=0$. The binary operation defined on $\mathcal{F}$ by $f \equiv g$ if and only if there exists $\alpha>0$ such that $f=\alpha \cdot g$ is an equivalence relation, with $c(f)=\{\alpha \cdot f, \alpha>0\}$ the coset of $f$. Now, denote by $\mathcal{F} / \equiv$ the quotient set of $\equiv$ and consider the mapping

$$
\begin{aligned}
\Psi: \mathcal{M}_{\text {shsm }} \longrightarrow \mathcal{F} / \equiv & \\
& m \longmapsto \Psi(m)=c(f), \quad \text { where } m=m_{f} .
\end{aligned}
$$

Then we get the following corollary.

Corollary 4.5 The mapping $\Psi$ defined by (4.5) is well defined and realizes an injection between the set of symmetric homogeneous strict monotone means, $\mathcal{M}_{\text {shsm }}$, and the set $\mathcal{F} / \equiv$.

Proof Due to Theorem 4.4, for every $m \in \mathcal{M}_{\text {shsm }}$, there exists $f \in \mathcal{F}$ such that $m=m_{f}$. To check that $\Psi$ is a map, consider two means such that $m_{1}=m_{2}$ and let $f_{1}, f_{2}$ be the two associated intrinsic functions, $m_{1}=m_{f_{1}}$ and $m_{2}=m_{f_{2}}$. We need to check that $c\left(f_{1}\right)=c\left(f_{2}\right)$. Since $m_{f_{1}}=m_{f_{2}}$, Theorem 2.4 implies that there exists $\alpha>0$ such that $f_{1}=\alpha \cdot f_{2}$, and so $c\left(f_{1}\right)=c\left(f_{2}\right)$.

To check the injectivity of $\Psi$, let $m_{1}$ and $m_{2}$ be two means such that $\Psi\left(m_{1}\right)=\Psi\left(m_{2}\right)$. This means that $c\left(f_{1}\right)=c\left(f_{2}\right)$, where $f_{1}, f_{2}$ are respectively the two associated intrinsic functions of $m_{1}$ and $m_{2}$. By the definition of the equivalence relation $\equiv, c\left(f_{1}\right)=c\left(f_{2}\right)$ implies that there exists $\alpha>0$ such that $f_{1}=\alpha \cdot f_{2}$, which again by Theorem 2.4 implies that $m_{1}=m_{2}$. Note here that $\Psi$ is not onto because for a given $f \in \mathcal{F}$, the mean $m_{f}$ is not necessarily symmetric. The proof is finished. 
Now, we will state some examples that illustrate our theoretical results and show the generality of our approach.

Example 4.1 Let $m=A$ be the arithmetic mean with its associate function $\phi(x)=(x+1) / 2$. Due to Lemma 4.3, the solution of the ODE (4.3) associated to $A$ looks like

$$
y(x)=\exp \int \frac{\phi^{\prime}(x)}{x-\phi(x)} d x=\exp \int \frac{1}{x-1} d x=\exp \ln |x-1|=|x-1|
$$

since $y(1)=0$. Following the choice in the proof of Lemma 4.3, we can choose $f(x)=x-1$. Now, by (2.3) we can check that

$$
m_{f}(a, b)=\frac{b}{a / b-1} \int_{1}^{a / b} x f^{\prime}(x) d x=\frac{b^{2}}{a-b}\left[\frac{x^{2}}{2}\right]_{1}^{a / b}=\frac{a+b}{2}=A(a, b) .
$$

Example 4.2 Now, consider $m=G$, the geometric mean whose associate function is $\phi(x)=\sqrt{x}$. Similarly to the previous example, we have

$$
y(x)=\exp \int \frac{\frac{1}{2 \sqrt{x}}}{x-\sqrt{x}} d x=\exp \int \frac{d u}{u(u-1)}
$$

where we make the change of variables $u=\sqrt{x}$. A classical integral computation, with $y(1)=0$, leads to $y(x)=\left|1-\frac{1}{\sqrt{x}}\right|$, and then we choose $f(x)=1-\frac{1}{\sqrt{x}}$. To check this, we can by (2.3) and a simple computation see that

$$
m_{f}(a, b)=\frac{b}{1-\sqrt{b / a}} \int_{1}^{a / b} x \cdot \frac{1}{2 x \sqrt{x}} d x=\frac{b \sqrt{a}}{\sqrt{a}-\sqrt{b}}[\sqrt{x}]_{1}^{a / b}=\sqrt{a b}=G(a, b) .
$$

Example 4.3 Let $m=H$ be the harmonic mean. Then

$$
\phi(x)=\frac{2 x}{x+1}, \quad \phi^{\prime}(x)=\frac{2}{(x+1)^{2}}, \quad x-\phi(x)=\frac{x(x-1)}{x+1} .
$$

The general solution of the ODE (4.3) is

$$
\begin{aligned}
y(x) & =\exp \int \frac{2}{x(x-1)(x+1)} d x=\exp \int\left(\frac{-2}{x}+\frac{1}{x-1}+\frac{1}{x+1}\right) d x \\
& =\exp (-2 \ln x+\ln |x-1|+\ln (x+1))=\frac{|x-1|(x+1)}{x^{2}}
\end{aligned}
$$

By the same arguments as before the associated intrinsic function $f$ to $H$ is given by

$$
\forall x>0, \quad f(x)=\frac{(x-1)(x+1)}{x^{2}}=1-\frac{1}{x^{2}} .
$$

To check that $m_{f}=H$, we can also use (2.3) with a routine integral computation.

Example 4.4 Let $m=L$ be the logarithmic mean. Then

$$
\phi(x)=\frac{x-1}{\ln x}, \quad \phi^{\prime}(x)=\frac{x \ln x-x+1}{x(\ln x)^{2}}, \quad x-\phi(x)=\frac{x \ln x-x+1}{\ln x}=(x \ln x) \phi^{\prime}(x) .
$$


Here, we have

$$
y(x)=\exp \int \frac{d x}{x(\ln x)}=\exp (\ln |\ln x|)=|\ln x|
$$

We then choose $f(x)=\ln x$ for all $x>0$. We can easily verify that $m_{f}=L$.

Example 4.5 Let $m=Q$ be the root-square mean. It is not hard to verify that the solution of ODE (4.3) is here given by

$$
y(x)=\exp \frac{1}{2} \int \frac{x}{x \sqrt{\frac{x^{2}+1}{2}}-\frac{x^{2}+1}{2}} d x
$$

Making the change of variables $t=x / \sqrt{1+x^{2}}$, an elementary computation leads to

$$
y(x)=\exp \int \frac{t}{(\sqrt{2} t-1)\left(1-t^{2}\right)} d t=\exp \int\left(\frac{\sqrt{2}}{\sqrt{2} t-1}+\frac{\sqrt{2}+1}{2(1-t)}+\frac{\sqrt{2}-1}{2(1+t)}\right) d t,
$$

which, after simple computation and appropriate reduction (with $y(1)=0$ and by the same reason as before), yields

$$
\forall x>0, \quad f(x)=\left(x \sqrt{2}-\sqrt{1+x^{2}}\right) \exp \left(\sqrt{2} \ln \left(x+\sqrt{1+x^{2}}\right)\right) .
$$

Of course, we have $m_{f}=Q$, but such a relationship is not easy to check here.

Example 4.6 Let $m=S$ be the square-root mean. We leave to the reader the routine task to see that the intrinsic function $f$ associated to $S$ is given by

$$
\forall x>0, \quad f(x)=\frac{\sqrt{x}-1}{(3 \sqrt{x}+1)^{1 / 3}}
$$

and to check that $m_{f}=S$.

The next example shows that the assumption ' $m$ is a monotone mean' in Theorem 4.4 is necessary.

Example 4.7 Let $m=C$ be the contra-harmonic mean. Of course, we cannot apply Theorem 4.4 since $C$ is not monotone. By Corollary 2.3, there is no $f \in C_{\uparrow}^{1}(0, \infty)$ such that $C=m_{f}$. We will check this by solving the ODE (4.3). In fact, here we have

$$
\phi(x)=\frac{x^{2}+1}{x+1}, \quad \phi^{\prime}(x)=\frac{x^{2}+2 x-1}{(x+1)^{2}}, \quad x-\phi(x)=\frac{x-1}{x+1}
$$

and the appropriate solution of the ODE (4.3) is given by

$$
y(x)=\exp \int \frac{x^{2}+2 x-1}{x^{2}-1} d x=\exp \int\left(1+\frac{1}{x+1}+\frac{1}{x-1}\right) d x=\left|x^{2}-1\right| e^{x}
$$


Following our choice, $f(x)=\left(x^{2}-1\right) e^{x}$. Theorem 4.4 does not confirm the relationship $m_{f}=C$. In fact, (2.4) implies

$$
(\forall a, b>0) \quad m_{f}(a, b)=a-\frac{b^{3}}{\left(a^{2}-b^{2}\right) e^{a / b}} \int_{1}^{a / b}\left(x^{2}-1\right) e^{x} d x .
$$

After twice integration by parts, we can easily see that

$$
m_{f}(a, b) \neq \frac{a^{2}+b^{2}}{a+b}=C(a, b) .
$$

Finally, we state the following example, which includes a lot of particular situations previously discussed.

Example 4.8 Let $p$ be a fixed real number, and let $m=D_{p}$ be the power difference mean defined in Example 2.2. Such a power mean, which is symmetric homogeneous strict monotone, is in turn a particular case of the so-called Stolarsky mean; see [17]. Its associate function $\phi_{p}$ satisfies

$$
\forall x>0, \quad \frac{\left(\phi_{p}\right)^{\prime}(x)}{x-\phi_{p}(x)}= \begin{cases}\frac{p x^{p-1}}{x^{p}-1} & \text { if } p \neq 0, \\ \frac{1}{x \ln x} & \text { if } p=0 .\end{cases}
$$

The associated intrinsic function $f_{p}$ is then given by

$$
\forall x>0, \quad f_{p}(x)= \begin{cases}x^{p}-1 & \text { if } p>0 \\ 1-x^{p} & \text { if } p<0 \\ \ln x & \text { if } p=0\end{cases}
$$

The particular cases $p=1, p=0, p=-2, p=-1$, and $p=-1 / 2$ were previously discussed; see Example 2.1.

Competing interests

The authors declare that they have no competing interests regarding the present manuscript.

Authors' contributions

Both authors worked in coordination. Both authors carried out the proof, read and approved the final version of the manuscript.

\section{Author details}

1Department of Mathematics, Science Faculty, Taibah University, P.O. Box 30097, Al Madinah Al Munawwarah, 41477,

Saudi Arabia. ${ }^{2}$ Department of Mathematics, Science Faculty, Moulay Ismail University, Meknes, Morocco. ${ }^{3}$ Mathematics

Department, INSAT, Carthage University, Tunis, Tunisia.

\section{Acknowledgements}

The authors would like to thank the anonymous referees for their comments and suggestions, which have been included in the final version of this manuscript.

Received: 20 May 2016 Accepted: 16 August 2016 Published online: 13 September 2016

\section{References}

1. Raïssouli, M: On an approach in service of mean-inequalities. J. Math. Inequal. 10(1), 83-99 (2016)

2. Sándor, J: On means generated by derivatives of functions. Int. J. Math. Educ. Sci. Technol. 28(1), 146-148 (1997)

3. Sándor, J: A note on the logarithmic mean. Am. Math. Mon. 123(1), 112 (2016)

4. Sándor, J, Toader, G: Some general means. Czechoslov. Math. J. 49(124), 53-62 (1999)

5. Toader, G, Sándor, J: Inequalities for general integral means. J. Inequal. Pure Appl. Math. 7(1), Article 13 (2006)

6. Raïssouli, M, Sándor, J: On a method of construction of new means, with applications. J. Inequal. Appl. 2013, 89 (2013) 
7. Bullen, PS: Handbook of Means and Their Inequalities, 2nd edn. Mathematics and Its Applications. Springer, Berlin (1987)

8. Neuman, E, Sándor, J: On the Schwab-Borchardt mean. Math. Pannon. 14(2), 253-266 (2003)

9. Neuman, E, Sándor, J: On the Schwab-Borchardt mean II. Math. Pannon. 17(1), 49-59 (2006)

10. Seiffert, HJ: Problem 887. Nieuw Arch. Wiskd. 11, 176 (1993)

11. Seiffert, HJ: Aufgabe 16. Wurzel 29,87 (1995)

12. Neuman, E: On some means derived from the Schwab-Borchardt mean. Math. Pannon. 8(1), 171-183 (2014)

13. Dragomir, SS: Two mappings in connection to Hadamard's inequalities. J. Math. Anal. Appl. 167, $42-56$ (1992)

14. Dragomir, SS, Mcandrew, A: Refinements of the Hermite-Hadamard inequality for convex functions. J. Inequal. Pure Appl. Math. 6(5), Article 140 (2005)

15. Dragomir, SS, Raïssouli, M: Iterative refinements of the Hermite-Hadamard inequality, application to the standard means. J. Inequal. Appl. 2010, Article ID 107950 (2010)

16. Yang, GS, Hong, MC: A note on Hadamard's inequality. Tamkang J. Math. 28(1), 33-37 (1997)

17. Stolarsky, KB: The power and generalized logarithmic means. Am. Math. Mon. 87, 545-548 (1980)

\section{Submit your manuscript to a SpringerOpen ${ }^{\odot}$} journal and benefit from:

- Convenient online submission

Rigorous peer review

- Immediate publication on acceptance

- Open access: articles freely available online

- High visibility within the field

- Retaining the copyright to your article 